\title{
WOOD SPECIES IDENTIFICATION BASED ON AN ENSEMBLE OF DEEP CONVOLUTION NEURAL NETWORKS
}

\author{
Tao $\mathrm{He}^{1}$, Shibiao $\mathrm{Mu}^{2}$, Houkui $Z_{\mathrm{Hou}^{1}}$, Junguo $\mathrm{Hu}^{1}$ \\ 1Zhejiang A\&F University \\ CHINA \\ ${ }^{2}$ Yinu Industrial \& Commercial College \\ CHINA
}

(Received March 2020)

\begin{abstract}
Our paper proposed an ensemble framework of combining three deep convolution neural networks (CNN). This method was inspired by network in network. Transfer learning used to accelerate training and deeper layers of network. Nine different $\mathrm{CNN}$ architectures were trained and evaluated in two wood macroscopic images datasets. After two times of 30 epochs training, our proposed network obtained $100 \%$ test rate in our dataset, which including 8 kinds of wood species and 918 images. The proposed method achieved $98.81 \%$ test recognition rate after three times training with 30 epochs in other dataset, which including 41 kinds of wood species and 11,984 images. Results showed that magnification macroscopic images can be instead of microscopic images in wood species identification, and our proposed ensemble of deep CNN can be used for wood species identification.
\end{abstract}

KEYWORDS: Wood identification, deep convolution neural networks, ensemble framework, macroscopic images.

\section{INTRODUCTION}

Wood is regarded as a renewable and environmental material (Yasar 2019). Different kinds of wood species are far from each other in use and price (Barmpoutis et al. 2018). It is important to develop a quick and accurate method to identify wood species, which not only leads to the proper utilization of woods, but also to the prevention of wood smuggling and the protection of several endangered tree species.

Wood researches focus on microstructure or macroscopic of the wood (Gong et al. 2019). So microscopic images and macroscopic images are used to identify wood species in machine 
learning. Microscopic images have two disadvantages: (1) For obtaining wood anatomical features (Al-Mefarrej and Suansa 2019), acquisition method is destructive, because wood samples must be sliced or flattened to expose three different planes: the transverse, radial, and tangential sections (Kobayashi et al. 2019). (2) The process is quite complex and laborious. Since the wood samples must be boiled to make it softer at first, then cut with a sliding microtone to a thickness, colored with (acridine red, chrysoidine, or astra blue), dehydrated in an ascending alcohol series and finally acquired the image from sheets of wood using a microscope (Filho et al. 2014). The complexity of the make procedure of wood slices used in the microscopic approach does not make it suitable for the use in the field, where one needs less expensive and more robust hardware. To overcome this problem some authors (Barmpoutis et al. 2018, Hu et al. 2016, Filho et al. 2014) have investigated the use of macroscopic images to identify forest species. Compared to the microscopic image, macroscopic image presents some significant loss of information related to specific features of the forest species, but it is easy to obtain and enough to distinguish wood species.

With the rapid development of information technology, image processing technology and machine learning technology have been widely used in wood classification. Traditional machine learning algorithms used in wood identification, which contain Linear discriminant analysis, Binary tree classification, Logical linear regression, K-order nearest neighbor classification, Bayesian classification, Support vector machine (Mallik et al. 2011). These above algorithms are based on image preprocessing and image feature extraction. In addition, most of the current computer automatic recognition systems of wood categories are based on the microstructure image of wood slices (Filho et al. 2014, Maruyama et al. 2018).

Deep learning as a branch of artificial intelligence has made a great progress in recently years. Convolutional neural network (CNN), as a technical direction of deep learning, has successfully made a great breakthrough in image classification (Krizhevsky et al. 2012). In this paper, an ensemble of deep CNN is applied to classification and recognition of wood species. Compared to the traditional wood recognition method, it has two advantages: (1) Traditional wood recognition technologies need to extract various features of wood, such as (heartwood and sapwood) color, axial parenchyma, wood ray, ring, conduit and wood texture (Sundaram et al. 2015, Baas et al. 2004). The quality of features extraction will influence the accuracy of wood recognition. There are many disadvantages in these methods, such as large amount of data operation, large amount of work and require domain experience in feature extraction and feature selection. However, deep $\mathrm{CNN}$ does not need features extraction by human. It requires very little engineering by hand, just inputting the original data directly, it can extract feature by itself, which avoid human operational bias will improve recognition accuracy. (2) Wood images are not completely normalized. Because environmental factors such as distance, height, angle and illumination often cause image scaling, rotation, blur and other changes, which increases the difficulty of recognition. Deep CNN insensitive to large irrelevant variations such as the background, pose, lighting and surrounding objects (Lecun et al. 2015), which reduce the external interference factors of wood image effectively.

One contribution of our work is proposed an effective ensemble of deep learning methodology, which is used to identification wood species. The deeper layers of network, the higher accuracy the model achieve in the same dataset (Szegedy et al. 2015, Simonyan and Zisserman 2015). Transfer learning is easy to build a deep layers model and leverage the feature extracting capability of the trained layers (Pan 2014). Transfer learning is used in our method, models are downloaded from Tensorflow (Abadi et al. 2016), which are pre-trained with ImageNet (Russakovsky et al. 2015). Inspired by network in network (Lin et al. 2014). A framework in framework is proposed in our method, the same network training three times as a small framework and sum of each prediction 
probability, three different frameworks are integrated as a framework, which add probability of each framework, finally sum all the predict probability to identification the wood species.

Nine kinds of popular deep learning models based on CNN for wood identification to compare test rate by transfer learning (Leonardo et al. 2019), including VGG16, Inception v3, ResNet50 v2, ResNet101 v2, ResNet152 v2, InceptionResNet v2, DenseNet121, DenseNet169, and DenseNet201. Three highest test accuracy models in the above models are selected and used in our ensemble framework, which are DenseNet121, DenseNet201 and InceptionResNet v2. The identification algorithms are trained, validated and tested in two wood datasets. One dataset contains 8 wood species consisting 918 macroscopic images created by our lab called Stereogram Wood Dataset (SWD), the other one called Forest Species Database-Macroscopic (FSD-M) ,which includes 41 kinds of wood species and 35,952 wood images (Filho et al. 2014). The ensemble algorithm gets higher accuracy than a single deep CNN in both datasets. The ensemble method achieves $100 \%$ test accuracy in SWD after two times of 30 epochs training, and $98.81 \%$ in FSD-M after three times of 30 epochs training which $1.04 \%$ higher than the best method proposed by Filho et al. 2014.

\section{MATERIAL AND METHODS}

\section{Datasets description}

Stereogram-wood images were acquired by stereo microscope connected with a computer. The stereo microscope using an Olympus DP70 connected with MD50 model which was manufactured by Guangzhou Mingmei, the microscope has 16 times maximum magnification. Eight kinds of wood species images were obtained in 8 times magnification. After obtaining the large cross-section of the sample wood images, cutting the images into small images with $224 \times 224$ pixels 24 bit RGB. Tab. 1 describes the eight wood species in the dataset, and selected sample images with 8 times magnification are shown as Fig. 1.

Tab. 1: Description of SWD.

\begin{tabular}{|c|l|c|}
\hline ID & \multicolumn{1}{|c|}{ Species } & Images \\
\hline 1 & Quercus acutissima Carr. & 108 \\
\hline 2 & Quercus variabilis B1. & 108 \\
\hline 3 & Celtis biondii Bl. & 108 \\
\hline 4 & Ulmus parvifolia Jacq. & 108 \\
\hline 5 & Sassafras tzumu (Hemsl.)Hemsl. & 108 \\
\hline 6 & Euodia rutaecarpa (Juss.)Benth & 162 \\
\hline 7 & Ailanthus altissima (Mill.)Swingle & 108 \\
\hline 8 & Meliosma angustifolia Franch. & 108 \\
\hline
\end{tabular}




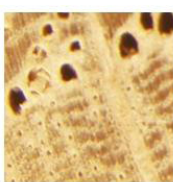

1

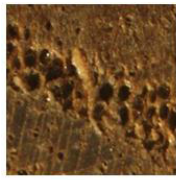

5

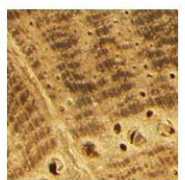

2

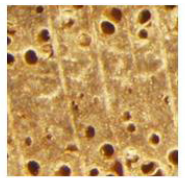

6

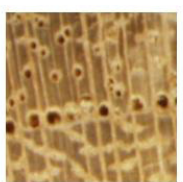

3

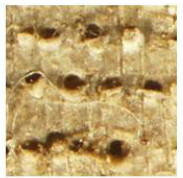

7

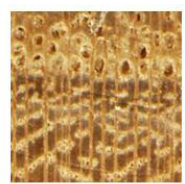

4

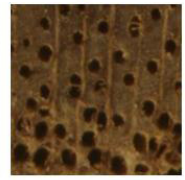

8

Fig. 1: Selected cross section of wood stereogram images (as described in Tab.1).

The wood macroscopic images dataset was collected using a Sony DSC T20 with the macro function activated. The resulting images were then saved in JPG format with no compression and a resolution of 3,264 $\times 2,448$ pixels. In total 2,942 macroscopic images had been acquired and carefully labeled by experts in wood anatomy. To enlarge the dataset, every image was clipped to 4 images, with $1120 \times 1120$ pixels. Tab. 2 describes the 41 wood species in the database, and sample images are shown as Fig. 2.

Tab. 2: Description of FSD-M.

\begin{tabular}{|c|l|c|c|l|c|}
\hline ID & \multicolumn{1}{|c|}{ Species } & Images & ID & \multicolumn{1}{|c|}{ Species } & Images \\
\hline 1 & Aspidosperma polyneuron & 212 & 22 & Cariniana estrellensis & 396 \\
\hline 2 & Araucaria angustifolia & 252 & 23 & Couratari sp. & 256 \\
\hline 3 & Tabebuia sp. & 172 & 24 & Carapa guianensis & 328 \\
\hline 4 & Cordia goeldiana & 396 & 25 & Cedrela fissili & 220 \\
\hline 5 & Cordia sp. & 252 & 26 & Melia azedarach & 184 \\
\hline 6 & Hura crepitans & 164 & 27 & Swietenia macrophylla & 348 \\
\hline 7 & Acrocarpus fraxinifolius & 192 & 28 & Brosimum paraense & 368 \\
\hline 8 & Hymenaea sp. & 300 & 29 & Bagassa guianensis & 384 \\
\hline 9 & Peltogyne sp. & 288 & 30 & Virola surinamensis & 204 \\
\hline 10 & Hymenolobium petraeum & 392 & 31 & Eucalyptus sp. & 380 \\
\hline 11 & Myroxylon balsamum & 144 & 32 & Pinus sp. & 396 \\
\hline 12 & Dipteryx sp. & 396 & 33 & Podocarpus lambertii & 172 \\
\hline 13 & Machaerium sp. & 224 & 34 & Grevilea robusta & 316 \\
\hline 14 & Bowdichia sp. & 268 & 35 & Balfourodendron riedelianum & 248 \\
\hline 15 & Mimosa scabrella & 204 & 36 & Euxylophora paraensis & 236 \\
\hline 16 & Cedrelinga catenaeformis & 312 & 37 & Micropholis venulosa & 232 \\
\hline 17 & Goupia glabra & 396 & 38 & Pouteria pachycarpa & 396 \\
\hline 18 & Ocotea porosa & 212 & 39 & Manilkara huberi & 232 \\
\hline 19 & Mezilaurus itauba & 376 & 40 & Erisma uncinatum & 248 \\
\hline 20 & Laurus nobilis & 344 & 41 & Vochysia sp. & 552 \\
\hline 21 & Bertholethia excelsa & 392 & & Total & $\mathbf{1 1 , 9 8 4}$ \\
\hline & & & &
\end{tabular}




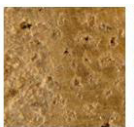

1
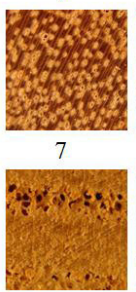

13

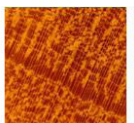

19

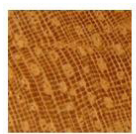

25

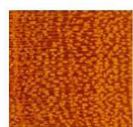

31

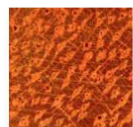

37

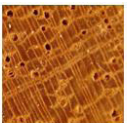

2

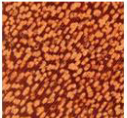

8

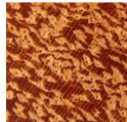

14

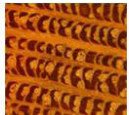

20

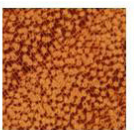

26

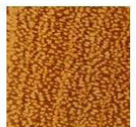

32

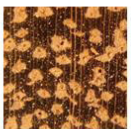

38

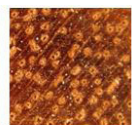

3

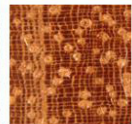

9

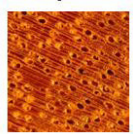

15

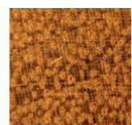

21

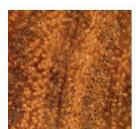

27

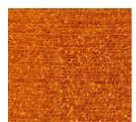

33

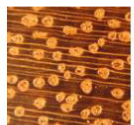

39

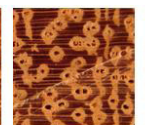

4

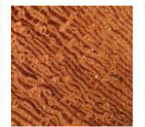

10

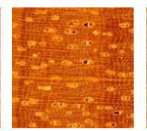

16

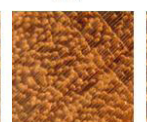

22

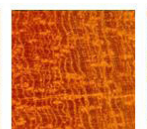

28

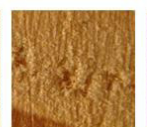

34

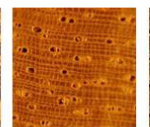

40

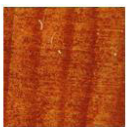

5

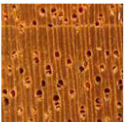

11

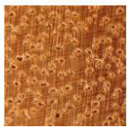

17

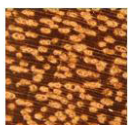

23

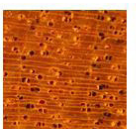

29

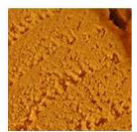

35

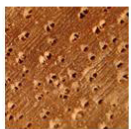

41

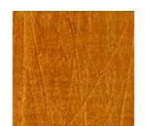

6

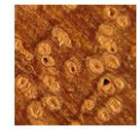

12

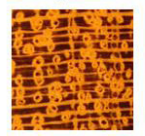

18

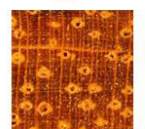

24

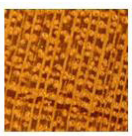

30

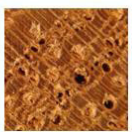

36

Fig. 2: Selected samples of FSD-M of 41 different kinds of wood (as described in Tab. 2).

\section{Experimental environment and data preprocess}

The experimental computer environment was $3.5 \mathrm{GHz}$ i7-7800X CPU, 32GB memory, 2 NVIDIA Geforce GTX $1080 \mathrm{Ti}$, windows 10 operating system. Python 3.7 and CUDA 10.2 were installed. Attribute to highly modular neural network library, Tensorflow 2.1.0-gpu was installed, which is a newest version of Tensorflow supporting GPU to accelerate computation.

Deep learning requires thousands of samples, so random augmentations were applied to compensate for lacking of insufficient training samples. A sequence of augmentation steps were defined in the augmentation process (Jalali et al. 2020). Data preprocess included resize image to the input size of the model, up and down flip, random crop of images, normalization the images to $0 \sim 1$, finally change each image to a tensor. The labels of images were turned to one-hot coding. Both datasets were applied the same strategy: $60 \%$ of images were shuffled random for training, $20 \%$ of images were for validation, and $20 \%$ of images were for testing. 


\section{Methodology}

\section{Transfer learning}

Transfer learning methods have been widely adopted in image classification and other fields due to their little sample sizes. Higher layers of representation amplify aspects of the input, which are important for discrimination and suppress irrelevant variations to classification (Lecun et al. 2015). Because layers of net are crucial to CNN, transfer learning was applied to deeper layers in our paper. Transfer learning retrained deep learning models and the wood species identification tasks were evaluated in terms of accuracy and efficiency.

Pre-trained models which had trained in other datasets, such as ImageNet (Deng et al. 2009) dropping its final classification layer as fixed feature extractor can learn complex features of the wood macroscopic images. All these learned layers were connected to a fully connected layer, a batch normal layer and a dropout layer, final layer with a dense to classify wood species.

Models were used to train on both wood macroscopic images datasets using transfer learning to compare with our proposed method. Nine kinds of popular deep learning models based on CNN for wood identification by transfer learning (Leonardo et al. 2019), including VGG16, Inception v3, ResNet50 v2, ResNet101 v2, ResNet152 v2, InceptionResNet v2, DenseNet121, DenseNet169, and DenseNet201. Information extracted from images by ResNet equal to extracted by Inception (McNeely-White et al. 2020). The use of residual connections seems to improve the training speed greatly, which is alone a great argument for their use (Szegedy et al. 2017). So, InceptionResNet was used in our network. Guo used two different layers of Resnet as a framework to train datasets, achieved a result of 0.917 on the test set, which is 0.046 higher than a single ResNet (Guo and Yang 2018). Doing to above conclusions, our proposed method integrated with InceptionResNet v2, DenseNet121, and DenseNet169 to identification wood species. Our method contains different layers of network and different networks.

\section{$V G G$}

VGG net is a CNN model proposed by Simonyan and Zisserman, which holds an architecture with very small $(3 \times 3)$ convolution filter to achieved the depth to $16-19$ weight layers. Max pooling handles reducing the size of the volume (down-sampling). Additionally, two fully connected layers each with 4096 nodes and a softmax classifier as shown in their work (Simonyan et al. 2015). VGG16 has a depth of 16 layers, which is adopted in our test.

\section{GoogLeNet Inception and InceptionResNet}

The "Inception" concept was first introduced in the GoogLeNet architecture by Szegedy et al. (2017). Now the latest version is Inception V4. This architecture combines the Inception architecture with residual connections, which aim being to accelerate the training of Inception networks.

The Inception module is made up of a pooling layer and convolution layers stacked together. The convolutions are of varied sizes of $1 \times 1,3 \times 3$ and $5 \times 5$. Another salient feature of the Inception module is the use of bottleneck layer which is a $1 \times 1$ convolution. The bottleneck layer helps in reduction of computation requirements. Additionally, there is pooling layer is used for dimension reduction within the module. InceptionResNet is a costlier hybrid Inception version, which combined with ResNet has significantly improved recognition performance (Szegedy et al. 2017). GoogLeNet Inception v3 and InceptionResNet v2 by using pre-trained weights from Tensorflow were performed in experiment. 
ResNet

ResNet model was first introduced by He et al. (He et al. 2016), which was a basis of their proposed model in ILSVRC 2015 and COCO 2015 classification challenge. Their model won the 1 st place with error rate of $3.57 \%$ in the ImageNet classification. ResNet is a network-innetwork architecture that relies on many stacked residual units. These residual units are the set of building blocks used to construct the network. A collection of residual unit's forms building blocks that leads to the ResNet architecture (He et al. 2016). The residual units are composed of convolution, pooling layers. The architecture is similar to the VGG net (Simonyan et al. 2015) consisting of $3 \times 3$ filters but ResNet, is about 8 times deeper than VGG network. This is attributed due to the usage of global average pooling rather than fully-connected layers. A further update of ResNet (He et al. 2016) was done to obtain more accuracy by updating the residual module to use identity mappings. ResNet50, ResNet101, ResNet152 with 50,101,152 layers downloaded with pre-trained weights from Tensorflow are used in our paper.

\section{DenseNet}

Huang et al. introduced a densely connected convolutional network architecture (Huang et al. 2017). To ensure maximum information flow between layers in the network, all layers are connected directly with each other in a feed-forward manner. For each layer, the feature maps of all preceding layers are used as inputs and its own feature maps are used as inputs into all subsequent layers. DenseNet alleviates the problem of the vanishing gradient problem and has substantially reduced number of parameters (Huang et al. 2016). For this task of wood species recognition, DenseNet models with 121, 169, 201 layers were used. The models were downloaded with pre-trained weights from Tensorflow.

\section{Our proposed ensemble framework of deep CNN model}

Deep CNN is currently one of the most popular models and has exhibited their great performance on many image classification problems (Kamilaris et al. 2018). The deeper layers of network will achieve higher accuracy to the same dataset (Szegedy et al. 2017), and combing different networks also get higher identification accuracy than single network (Guo et al. 2018). So, an effective deep CNN model for the identification of wood species is proposed in our paper, which is also inspired by network in network(Lin et al. 2014). Because of Resnet can deeper network easily, InceptionResNet combining the advantages of Inception and ResNet, and different layers of network also improve the identification accuracy. The ensemble model integrated InceptionResNet v2, ResNet121, and ResNet201. Fig. 3 presents the framework of our proposed model.

Images in both wood macroscopic datasets were reshaped to the size of $224 \times 224$ (wide and height) in this experiment. The above nine pre-trained models were downloaded from Tensorflow, which had pre-trained with ImageNet. By dropping the final classification layer as fixed feature extractor to learn complex features from wood images training dataset. All these learned layers were connected to a fully connected layer with ReLU (Rectified linear unit, ReLU) activation, then added a batch normal layer which can increase the speed of learning and the overall classification accuracy, and sequenced with a dropout layer to avoid over-fitting, finally with a dense layer to identify wood species. Inspired by network in network (Lin et al. 2014). A framework in framework was proposed in our method, the same network training three times as a small framework and summed each prediction probability, three different frameworks were integrated as a framework, which added probability of each framework, finally summed all the predict probability to identification the wood species. 


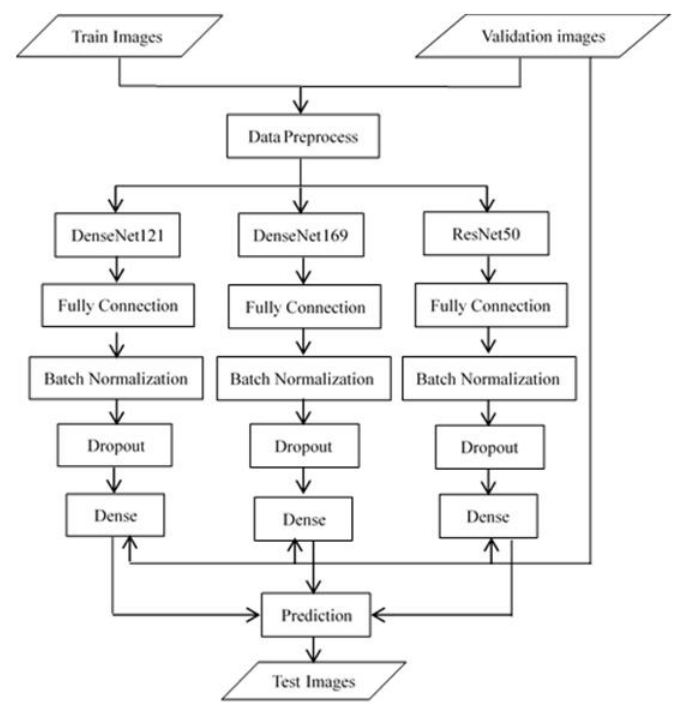

Fig. 3: Ensemble framework of deep CNN method.

Validation dataset was used to adjust parameters of network adjusted when training. The model evaluated based on cross-entropy loss and accuracy on the test dataset. Test dataset was used to evaluate the models identification accuracy. After training and validating with above nine kinds of CNN, used the network models to predict the probability labels of test images, adding three kind of CNN's predict labels, finally got the total probability labels, the maximum probability label as the identification label.

Following definitions of the recognition rate was used. Let $\mathrm{B}$ be a test set with $\mathrm{N}_{\mathrm{B}}$ images, $\mathrm{Acc}_{\mathrm{Rec}}$ represents as test accuracy of identification. If the recognition system classifies correctly $\mathrm{N}_{\mathrm{Rec}}$, then

$$
A c c_{R e c}=\frac{N_{R e c}}{N_{B}} \times 100 \%
$$

\section{RESULTS AND DISCUSSION}

\section{Training}

For every experiment, accuracy metric was adopted for evaluation of the models. The hyperparameters were standardized on all the networks. All the network models were trained using Stochastic Gradient Descent (SGD), which runs faster and converges easily (He et al. 2016). Batch Normalization technique and ReLU activation function (Glorot et al. 2011) were applied in all the experiments. Because of GPU memory constraints, batch size of 16 was used. The learning rate was set to 0.001 for all networks. In order to relieve the problem of little data, data augmentations were done to all networks including up and down flip, random crop of images. Nine different kinds of CNN were trained in the both dataset, and saved the model weight parameters. 


\section{Results of the experiments}

In our study, an assessment of the appropriateness of state-of-the-art deep CNN for the task of wood species identification using images was done. VGG16, Inception v3, ResNet50 v2, ResNet101 v2, ResNet152 v2, InceptionResNet v2, DenseNet121, DenseNet169, and DenseNet201 and our proposed ensemble deep convolutional neural network were trained and tested. In our paper, all models were trained 1 to 5 times, and trained for 30 epochs every time in both datasets. The average test identification accuracy of different train times of each models are shown in Tab. 3 and Tab. 4.

Tab. 3: Test recognition rates of various deep CNN learning algorithms in SWD.

\begin{tabular}{|l|c|c|c|c|c|}
\hline \multirow{2}{*}{\multicolumn{1}{|c|}{ Model }} & \multicolumn{5}{c|}{ Train times (\%) } \\
\cline { 2 - 6 } & 1 & 2 & 3 & 4 & 5 \\
\hline VG16 & 13.59 & 37.23 & 22.28 & 54.76 & 60.22 \\
\hline Inception v3 & 86.96 & 91.85 & 91.85 & 93.21 & 95.33 \\
\hline ResNet50 v2 & 86.96 & 85.05 & 88.41 & 91.30 & 85.54 \\
\hline ResNet101 v2 & 70.65 & 14.67 & 19.57 & 91.85 & 47.07 \\
\hline ResNet152 v2 & 80.43 & 14.95 & 19.57 & 82.07 & 79.67 \\
\hline InceptionResNet v2 & 96.20 & 97.55 & 91.85 & 84.92 & 96.09 \\
\hline DenseNet121 & 98.37 & 97.01 & 90.94 & 90.22 & 90.76 \\
\hline DenseNet169 & 97.28 & 71.11 & 75.56 & 87.78 & 75.56 \\
\hline DenseNet201 & 94.02 & 75.82 & 94.38 & 94.57 & 94.35 \\
\hline Ensemble of Deep CNN & 98.91 & 100.00 & 100.00 & 100.00 & 100.00 \\
\hline
\end{tabular}

Tab. 4: Test recognition rates of various deep CNN learning algorithms in FSD-M.

\begin{tabular}{|l|c|c|c|c|c|}
\hline \multirow{2}{*}{\multicolumn{1}{|c|}{ Model }} & \multicolumn{5}{c|}{ Train times (\%) } \\
\cline { 2 - 6 } & 1 & 2 & 3 & 4 & 5 \\
\hline VG16 & 53.81 & 53.81 & 81.50 & 77.48 & 87.07 \\
\hline Inception v3 & 95.17 & 95.17 & 73.56 & 93.06 & 93.10 \\
\hline ResNet50 v2 & 84.79 & 84.79 & 88.24 & 89.98 & 90.26 \\
\hline ResNet101 v2 & 70.26 & 70.26 & 66.37 & 67.14 & 79.71 \\
\hline ResNet152 v2 & 45.34 & 45.34 & 57.82 & 74.26 & 65.40 \\
\hline InceptionResNet v2 & 95.81 & 95.81 & 96.67 & 95.60 & 98.19 \\
\hline DenseNet121 & 92.60 & 76.21 & 86.57 & 95.19 & 95.48 \\
\hline DenseNet169 & 92.94 & 88.49 & 89.40 & 89.90 & 93.78 \\
\hline DenseNet201 & 90.38 & 93.47 & 92.52 & 92.76 & 93.64 \\
\hline Ensemble of Deep CNN & 96.98 & 97.66 & 98.81 & 98.85 & 99.46 \\
\hline
\end{tabular}

Tab. 3 and Tab. 4 have shown as follows: Our proposed ensemble of deep CNN obtained the highest test accuracy among the different networks. The accuracy achieved $100.00 \%$ in our dataset. After three times train, test accuracy got $98.81 \%$, which was $1.04 \%$ higher than the proposed method by Filho (Filho et al. 2014), and a confusion matrix is presented in Tab. 5 for each wood species in FSD-M. 
Tab. 5: Confusion matrix for FSD-M (numbers in brackets is mistaken predict label).

\begin{tabular}{|c|c|c|c|c|c|}
\hline \multirow{2}{*}{ ID } & \multicolumn{2}{|c|}{ Number of test images } & \multirow{2}{*}{ ID } & \multicolumn{2}{c|}{ Number of test images } \\
\cline { 2 - 3 } \cline { 6 - 6 } & Classification & Misclassification & & Classification & Misclassification \\
\hline 1 & 53 & 0 & 22 & 68 & $2(23)$ \\
\hline 2 & 77 & $1(17)$ & 23 & 37 & 0 \\
\hline 3 & 22 & $1(22)$ & 24 & 4 & 0 \\
\hline 4 & 1 & $1(37)$ & 25 & 65 & $3(26)$ \\
\hline 5 & 42 & 0 & 26 & 73 & 0 \\
\hline 6 & 56 & 0 & 27 & 26 & 0 \\
\hline 7 & 45 & $1(27)$ & 28 & 67 & $1(37)$ \\
\hline 8 & 73 & 0 & 29 & 5 & 0 \\
\hline 9 & 77 & $9(37)$ & 30 & 39 & $1(7)$ \\
\hline 10 & 42 & 0 & 31 & 54 & 0 \\
\hline 11 & 93 & 0 & 32 & 73 & 0 \\
\hline 12 & 52 & 0 & 33 & 33 & 0 \\
\hline 13 & 74 & 0 & 34 & 76 & 0 \\
\hline 14 & 7 & 0 & 35 & 47 & 0 \\
\hline 15 & 84 & $1(14)$ & 36 & 55 & $1(37), 2(38)$ \\
\hline 16 & 49 & 0 & 37 & 38 & $1(28)$ \\
\hline 17 & 59 & $1(3), 1(33)$ & 38 & 2 & $1(29)$ \\
\hline 18 & 46 & 0 & 39 & 47 & $1(40)$ \\
\hline 19 & 41 & 0 & 40 & 47 & 0 \\
\hline 20 & 67 & $3(15), 1(28)$ & 41 & 7 & 0 \\
\hline 21 & 76 & 0 & Total & $\mathbf{1 9 9 9}$ & $\mathbf{2 7}$ \\
\hline
\end{tabular}

As indicated by the Tab. 5, nine test images misclassification of wood species happened in the same family, and 27 test images misclassification in 2,026 test images. It illustrates that our proposed method is able to deal with the great intra-class variability presented by the forest species.

\section{CONCLUSIONS}

In this paper, an ensemble framework of deep CNN spired by network in network is introduced into the field of wood recognition, and a framework of wood feature extraction and recognition is constructed by deep CNN. The deep CNN has two advantages in the processing of wood macroscopic images: (1) Because there is no feature extraction step, the two-dimensional images are read directly into the network, which reduces the difficulty of image preprocessing; (2) local field and weight sharing technology greatly reduces the amount of parameters and the complexity of the algorithm. Traditional wood recognition technology has many problems, such as large number of images, more training data, time-consuming, etc., while deep CNN can better overcome the above shortcomings, and avoid the process of image extraction and classification, reducing the demand of artificial expertise.

From our experiment, some conclusions are obtained as follow: The ensemble of deep CNN integrates the advantages of various models which inspired by network in network and makes it achieve higher accuracy than single model. Mixed many times of train model parameters weight 
improve the identification accuracy. The transfer learning makes the data train more easily.

Compared to the microscopic image, macroscopic image presents some significant loss of information related to specific features of the forest species, but it is easy to obtain and enough to distinguish wood species by our proposed ensemble of deep CNN. The performance of our method in SWD is better than in FSD-M. Macroscopic images are captured in eight times magnification in SWD, but we do not know which times magnification in FSD-M. If the images are captured by less than eight times magnification, then we can get follow inclusion: higher magnification of the wood macroscopic images will improve wood species recognition, because more details help our proposed ensemble of deep CNN to identify the wood species.

The wood recognition system constructed in this paper has a high recognition rate for wood cross-section stereo images, and the proposed ensemble of deep CNN recognition rate of 8 kind of wood species in eight times magnification macroscopic image reaches $100 \%$, which are trained more than two times, and trained 30 iterations every time. Even though the performance of the architecture is good, further research needs to be done to improve on the computational time, and which magnification images is best for wood identification is also to be considered.

\section{ACKNOWLEDGMENTS}

This work was supported in part by the National Natural Science Foundation of China under Grant 31971493, Zhejiang Provincial Natural Science Foundation of China under Grant LY19F020048 and Zhejiang Provincial Key Laboratory of Forestry Intelligent Monitoring and Information Technology. The authors have no conflict of interest to declare.

\section{REFERENCES}

1. Abadi, M., Barham, P., Chen, J., Chen, Z., Davis, A., Dean, J., Devin, M., Ghemawat, S., Irving, G., Isard, M., Kudlur, M., Levenberg, J., Monga, R., Moore, S., Murray, D.G., Steiner, B., Tucker, P., Vasudevan, V., Warden, P. , Wicke ,M., Yu,Y., Zheng, X., 2016: Tensor flow: A system for large-scale machine learning. Proceedings of the $12^{\text {th }}$ USENIX Symposium on operating systems design and implementation, OSDI,16, Pp 265-283.

2. A1-Mefarrej, H.A., Suansa, N.I., 2019: Trait-based ecology using conduits comparison approach. Wood Research 64(6): 1055-1064.

3. Baas, P., Blokhina, N., Fujii, T., Gasson, P.E., Grosser, D., Heinz, I., Ilic, J., Xiaomei, J., Miller, R., Newsom, L.A., Noshiro, S., Richter, H.G., Suzuki, M., Terrazas, T., Wheeler, E., Wiedenhoeft, A., 2004: IAWA List of microscopic features for softwood identification. IAWA Journal 25(1): 1-70.

4. Barmpoutis, P., Dimitropoulos, K., Barboutis, I., Grammalidis, N., Lefakis, P., 2018: Wood species recognition through multidimensional texture analysis. Computers and Electronics in Agriculture 144: 241-248.

5. Deng, J., Dong, W., Socher, R., Li, L. jia, Li, K., Fei-fei, L., 2009: ImageNet: a large-scale hierarchical image database. IEEE Computer society conference on computer vision and pattern recognition (CVPR 2009): 20-25.

6. Filho, P.L.P., Oliveira, L.S.,Nisgoski, S., Britto, A.S., 2014: Forest species recognition using macroscopic images. Machine Vision and Applications 25: 1019-1031.

7. Gong, L., Zhang, Q., Liang, H., Ren, S., Wang, J., 2019: Mechanical properties and microstructure of Zabelia biflora. Wood Research 64(3): 423-436. 
8. Guo, S., Yang, Z., 2018: Multi-Channel-ResNet: An integration framework towards skin lesion analysis. Informatics in Medicine Unlocked 12: 67-74.

9. He, K., Zhang, X., Ren, S., Sun, J., 2016: Deep residual learning for image recognition. Proceedings of the IEEE Computer society conference on computer vision and pattern recognition 2016 Dec: 770-778.

10. Hu, S., Li, K., Bao, X., 2016: Wood species recognition based on SIFT keypoint histogram. In: Proceedings 8th International congress on image and signal processing (CISP), Pp 702-706.

11. Huang, G., Liu, Z., Van Der Maaten, L., Weinberger, K.Q. 2017: Densely connected convolutional networks. Proceedings - $30^{\text {th }}$ IEEE Conference on computer vision and pattern recognition, CVPR, Pp 4700-4708.

12. Jalali, A., Lee, M., 2020: High cursive traditional Asian character recognition using integrated adaptive constraints in ensemble of DenseNet and Inception models. Pattern Recognition Letters 131: 172-177.

13. Kamilaris, A., Prenafeta-Boldú, F. X., 2018: Deep learning in agriculture: A survey. Computers and Electronics in Agriculture 147(1): 70-90.

14. Kobayashi, K., Hwang, S. W., Okochi, T., Lee, W. H., Sugiyama, J., 2019: Non-destructive method for wood identification using conventional X-ray computed tomography data. Journal of Cultural Heritage 38: 88-93.

15. Krizhevsky, A., Sutskever, I., Hinton, G.E., 2012: ImageNet classification with deep convolutional neural networks. Advances in Neural Information Processing Systems 25(2): $1-9$.

16. Lecun, Y., Bengio, Y., Hinton, G., 2015: Deep learning. Nature 521: 436-444.

17. Leonardo, M.M., Carvalho, T.J., Rezende, E., Zucchi, R., Faria, F.A., 2019: Deep feature-based classifiers for fruit fly identification (Diptera: Tephritidae). Proceedings - 31 Conference on graphics, patterns and images, SIBGRAPI 2018: 41-47.

18. Lin, M., Chen, Q. Yan, S., 2014: Network in network. $2^{\text {nd }}$ International conference on learning representations (ICLR). Conference track proceedings, $10 \mathrm{pp}$.

19. Mallik, A., Tarrío-Saavedra, J., Francisco-Fernández, M., Naya, S., 2011: Classification of wood micrographs by image segmentation. Chemometrics and Intelligent Laboratory Systems 107(2): 351-362.

20. Maruyama, T. M.,Oliveira, L. S.,Britto, A. S.,Nisgoski, S., 2018: Automatic classification of native wood charcoal. Ecological Informatics 46: 1-7.

21. McNeely-White, D., Beveridge, J.R., Draper, B.A., 2020: Inception and ResNet features are (almost) equivalent. Cognitive Systems Research 59: 312-318.

22. Pan, S.J., 2014: Transfer learning. Data classification: Algorithms and applications ,IBM T. J. Watson Research Center Yorktown Heights, New York, USAIBM T. J. Watson Research CenterYorktown Heights, New York, USA, Pp 537-570.

23. Russakovsky, O., Deng, J., Su, H., Krause, J., Satheesh, S., Ma, S., Huang, Z., Karpathy, A., Khosla, A., Bernstein, M., Berg, A.C., Fei-Fei, L., 2015: ImageNet large scale visual recognition challenge. International Journal of Computer Vision 115(3): 211-252.

24. Simonyan, K., Zisserman, A., 2015: Very deep convolutional networks for large-scale image recognition. $3^{\text {rd }}$ International conference on learning representations (ICLR). Conference track proceedings, Corrnel University $14 \mathrm{pp}$.

25. Sundaram, M., Abitha, J., Mal Mathan Raj, R., Ramar, K., 2015: Wood species classification based on local edge distributions. Optik 126: 2884-2890. 
26. Szegedy, C., Ioffe, S., Vanhoucke, V., Alemi, A.A., 2017: Inception-v4, inception-ResNet and the impact of residual connections on learning. $31^{\text {st }}$ AAAI Conference on artificial intelligence, AAAI 2017: Pp 4278-4284.

27. Szegedy, C., Liu, W., Jia, Y., Sermanet, P., Reed, S., Anguelov, D., Erhan, D., Vanhoucke, V., Rabinovich, A., 2015: Going deeper with convolutions. Proceedings of the IEEE Computer society conference on computer vision and pattern recognition, Pp 1-9.

28. Yasar, S.S., 2019: Effects of wood preservatives on the combustion process and combustion quality of wood. Wood Research 64(1): 59-71.

\author{
Tao He, Houkui Zhou*, Junguo Hu* \\ School of Information EngineER \\ Zhejiang A\&F University \\ Lin'AN, ZheJIANG 3IIJOO \\ CHINA
}

*Corresponding authors: zhouhk@zju.edu.cn and hujunguo@zafu.edu.cn

\author{
Shibiao Mu \\ Yiwu Industrial \& Commercial College \\ Yiwu, Zhejiang 322300 \\ China
}


